\title{
Forecasting the Unknown Dynamics in NN3 Database Using a Nonlinear Autoregressive Recurrent Neural Network
}

\author{
E. Safavieh, S. Andalib, and A. Andalib
}

\begin{abstract}
In this paper, a nonlinear autoregressive (NAR) recurrent neural network is used for the prediction of the next 18 data samples of each time series in a set of 11 unknown dynamics in NN3 Database. The models are built on the reconstructed state spaces of data and no other domain knowledge is available to be used. Here, we clarify that the employed method is in part similar to a superior subclass of recurrent neural network, namely the nonlinear autoregressive model with exogenous inputs (NARX). Using the extensive available research about NARX networks, we briefly explain that our model is preferred to the both non-recursive and even other recurrent predictors, because of its intrinsic ability for learning long term dependencies in time series. As the desired values of the predicted time series are not available yet, no analysis have been performed on the presented results.
\end{abstract}

\section{INTRODUCTION}

$\mathrm{I}_{\mathrm{r} e m}^{\mathrm{s}}$ $\mathrm{N}$ time series prediction, we wish to build a model that is responsible for generation of a given time series. However, when the underlying dynamics is affected by a set of explanatory variables, which are not known, the model that captures the dynamics cannot be identified directly. In these cases, a proper mapping of the observable output of the unknown dynamical system may be helpful for the prediction of the time series at hand. Identification of this mapping is discussed in the theory of dynamic reconstruction [1]. Using the result of this theory the analysis, e.g. prediction, of the observable time series is possible. This is valuable if the evolution of the points in the reconstructed state space tracks that of the unknown dynamics in the original state space. Under some conditions, Takens delay-embedding theorem [2] introduces a diffeomorphic map (one to one deferential mapping) as the result of dynamic reconstruction theory. According to this theory, with estimating two parameters of embedding dimension $D_{E}$ and normalized embedding delay $\tau$, and constructing a predictive mapping, dynamic reconstruction is achieved. The reconstruction consists of two steps: first, a delay line should be designed to latch the information for $D_{E}$ points, and after that, a predictor that identifies the unknown mapping must be trained. This step is the heart of dynamic modeling.

Various nonlinear predictors are employed for the time

Manuscript received January 31, 2007. This work is supported in part by Sepanta Robotics \& AI Research Foundation (SRRF).

E. Safavieh, S. Andalib, and A. Andalib are with the SRRF, Tehran, 15757-18616, Iran (e-mail: e.safavieh@srrf.net, s.andalib@srrf.net, a.andalib@ srrf.net). series prediction task, including regression- and neural network-based algorithms. A review on some common methods for prediction of a chaotic time series, namely electricity price is presented in our recent work [3]. As it is described in [3], in many time series there is a very important characteristics, which is known as long-term dependencies, meaning that the temporal contingencies presented in the input/out sequences span long intervals. We have shown in[3], [4] that this is the case for financial time series and electricity price historical data.

It is mentioned in [5] that recurrent neural networks (RNNs) may be considered as a good choice for mapping input sequences to output sequences. In contrast, static systems, i.e. those with no recurrent connection, even if they include some lagged values of input data, have a finite impulse response and have difficulties with respect to RNN to store information for an indefinite time [5]. However, even simple recurrent networks, e.g. Elman RNN, are not ideal approaches for learning long-term dependencies because of the problem of vanishing gradient, meaning that under special condition given in section II, the fraction of error gradient due to information $n$ time steps in the past exponentially decreases as $n$ increases.

To tackle the problem of vanishing gradient, a class of recurrent neural networks, called nonlinear autoregressive model with exogenous inputs (NARX) is proposed [6], which has various advantages over simple recurrent networks. Not only has the NARX model less sensitivity to long-term dependencies [6], but also it has a very good learning capability and generalization performance [7].

It should be mentioned that NARX is different from a popular Auto Regressive (AR) model [8] which performs a simple linear transformation of the visited values of the time series. As it is mentioned in section II, the NARX is a nonlinear model which estimates the next values of the time series based on its last outputs instead of the actual measurements as is used in ARIMA models. Furthermore, the NARX uses a nonlinear structure, e.g. a neural network, for estimating the model's parameters. In contrast, the coefficients of a simple AR model are estimated using simple statistical methods like least squares estimation. Therefore, a NARX model enjoys a better generalization capability.

In this paper, we introduce the NARX model to use the research history that proves the advantages of this model over static and even simple recurrent structures. However, 
the time series that we are going to challenge in this paper are not multidimensional, in the sense that for each time series there is not any other explanatory data with values corresponding to the original one. Therefore, we are obligated to employ a simplified NARX network known as NAR. However, as the advantages of NARX are due to the feedback structure of this model, we are still interested in using a nonlinear autoregressive model without any exogenous inputs.

In this paper, we have employed aforementioned NAR model to forecast a set of 11 time series from the dataset of NN3 forecasting competition. As a preprocessing step, we first calculate some characteristics of these time series based on Takens' embedding theorem and the theory of dynamic reconstruction. The reconstructed state space is then used to generate 18 -step ahead forecasts.

This paper is organized as follows. In the next section, we briefly review the architecture of NAR in the framework of NARX recurrent neural network and enumerate its advantages over other recurrent structures. In section III, we review the Takens' embedding theory. In section IV, we present the reconstructed dynamics of time series in NN3 database. In section $\mathrm{V}$, we implement the methods explained in section II regarding the reconstructed state space addressed in section IV. Finally, the numerical results are presented in section VI.

\section{NARX RECURRENT NEURAL NETWORK}

A NARX model is a class of discrete-time nonlinear autoregressive systems, which has endogenous inputs as well as exogenous inputs, and can be stated as:

$$
\begin{array}{r}
\hat{y}(t+1)=f\left(y(t), \cdots, y\left(t-D_{y}\right),\right. \\
\left.u(t), \cdots, u\left(t-D_{u}\right)\right)+\varepsilon_{t}
\end{array}
$$

here $\{y(t)\}$ is the time series of interest that should be predicted and $\{u(t)\}$ is another time series with terms associated with that of $\{y(t)\}$. The terms $u(t), \cdots, u\left(t-D_{u}\right)$ are the exogenous inputs and may be produced with an input delay line with memory of order $D_{u}$. Similarly $y(t), \cdots, y\left(t-D_{y}\right)$ are the endogenous inputs and may be produced with a delay line memory of order $D_{y} \cdot f$ is some nonlinear function, e.g. a multi layer perceptron (MLP), that estimates the next value of $\{y(t)\}, \hat{y}(t+1)$ and $\varepsilon_{t}$ denotes the additive noise of the estimation. The architecture of a NARX recurrent neural network is shown in Fig. 1. The first layer of the MLP network consists of $D_{u}+D_{y}$ buffer neurons, corresponding to the outputs of the two delay lines.

Considering the above architecture, we define the cost function $E$ at the time $t$ as:
$E_{t}=1 / 2(\hat{y}(t)-y(t))^{2}$

We may mathematically explain the effect of vanishing

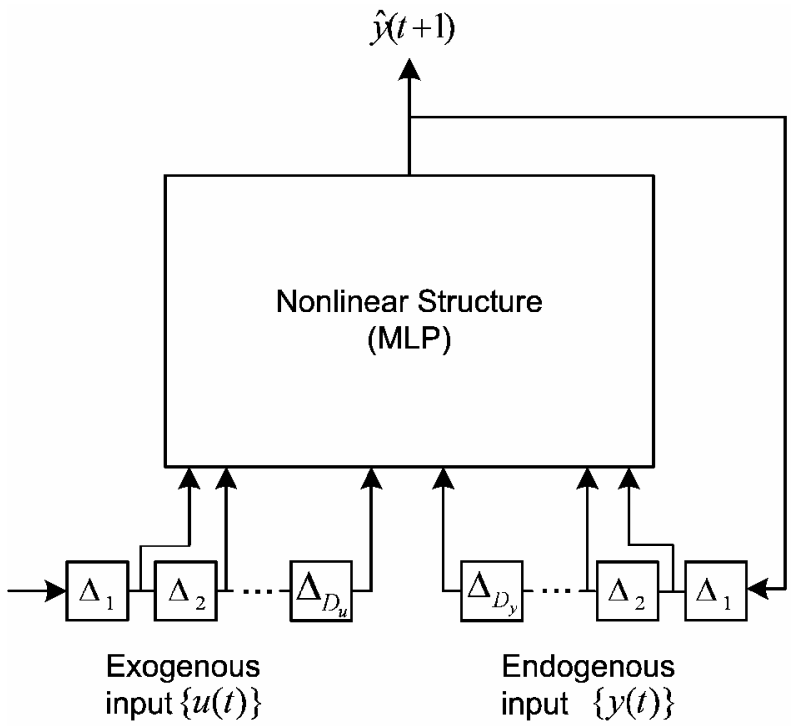

Fig. 1. A NARX recurrent neural network.

gradient on the derivatives of $E_{t}$ with respect to the weight vector $\mathbf{w}$ :

$$
\begin{aligned}
\frac{\partial E_{t}}{\partial \mathbf{w}} & =(\hat{y}(t)-y(t)) \frac{\partial \hat{y}(t)}{\partial \mathbf{w}} \\
& =(\hat{y}(t)-y(t)) \sum_{v \prec t} \frac{\partial \hat{y}(t)}{\partial \hat{y}(v)} \frac{\partial \hat{y}(v)}{\partial \mathbf{w}}
\end{aligned}
$$

It has been shown that if a network is to latch the information robustly, i.e. if it is to store information for a long period of time in the presence of noise, then for a term with $v<<t,|\partial \hat{y}(t) / \partial \hat{y}(v)| \rightarrow 0$ [5]. In this condition, the gradient decays exponentially [6], meaning that there is not any chance for the terms that are far from $t$ to change the weights in such a way that allow the network's state to jump to a better basin of attraction.

The above scenario comes true for all recurrent structures. However, one can postpone vanishing of gradient in NARX recurrent neural networks with increasing the number of delays in the output delay line of this architecture [6]. As it may be seen in Fig. 1, the output delay line of NARX networks, as jump-ahead connections, provides shortcuts for propagating gradient information more efficiently when the network is unfolded in time. In an unfolded recurrent neural network, the hidden units from the pervious states are considered as an additional set of inputs. This property makes NARX a proper tool for modeling dynamics that exhibit long-term dependencies.

As it is mentioned in section I, the NARX which is used in this paper does not have any exogenous inputs. 


\section{EMBEDDING THEORY AND DYNAMIC RECONSTRUCTION}

By the results of Takens' embedding theorem [9] and the theory of dynamic reconstruction [1], it is able to reconstruct the dynamics of interest, which were initially unobservable due to various, and maybe unknown parameters that effect on them.

Takens' theorem implies that by the means of two parameters of embedding dimension $D_{E}$ and normalized embedding delay $\tau$, it is guaranteed that the evolution of the points in the new state space follows that of the original state space. In this way the analysis, e.g. prediction of the new state space is fruitful.

Suppose the time series $\{y(t)\}$ as the observable output of the unknown dynamical system $\{x(t)\} ;\{y(t)\}$ may be defined as follows:

$$
y(t)=g(x(t))
$$

where $g(\cdot)$ is a scalar-valued function.

According to Takens' theorem, we can define $D$ dimensional reconstructed dynamics $\left\{\mathbf{y}_{R}(t)\right\}$ by the following equation:

$$
\begin{gathered}
\mathbf{y}_{R}(t)=[y(t), y(t-\tau), \ldots, y(t-(D-1) \tau], \\
D \geq 2 d+1
\end{gathered}
$$

where $d$ is the state space dimension of the unknown dynamics.

As it is mentioned above, the evolution of the points $\mathbf{y}_{R}(t) \rightarrow \mathbf{y}_{R}(t+1)$ tracks that of the unknown dynamics $x(t) \rightarrow x(t+1)$. So to challenge a problem concerned with the prediction of the time series $\{x(t)\}$, it is fruitful to predict the time series $\{y(t)\}$. This can be satisfied by a nonlinear model of $f: \mathfrak{R}^{D} \rightarrow \mathfrak{R}^{1}$, which performs the following mapping:

$$
\hat{y}(t+1)=f\left(\mathbf{y}_{R}(t)\right)
$$

where $\hat{y}(t+1)$ is the one-step ahead predicted value of the time series $\{y(t)\}$.

Equation (6) may be extended to $\tau$-step mapping with a different model of $f$ :

$$
\hat{y}(t+\tau)=f\left(\mathbf{y}_{R}(t)\right)
$$

There are many methods for estimating the embedding parameters. One of the methods for choosing the embedding delay is to choose the first point of autocorrelation function that goes bellow zero zone [10]. In [11] it is selected as the reciprocal of the highest relevant frequency of the time series. It is proposed in [12] to use the information dimension, which is a kind of fractal dimensions, as the time delay. However, one of the most reliable methods is presented by Fraser [13]. Due to this method known as average mutual information (AMI), the normalized embedding delay $\tau$ is heuristically set to the value in which the mutual information between $y(t)$ and $y(t-\tau)$ attains its first minimum. This way, the values $y(t)$ and $y(t-\tau)$ are essentially independent of each other in the sense that they may serve as two coordinates of the reconstructed space. However, they are not so independent as to have no correlation with each other.

There are also some methods for choosing embedding dimension $D_{E}$ such as correlation dimension and the Integral Local Deformation (ILD) algorithm [14]. One of the best and simplest methods is presented in [15]. According to this method, the minimum acceptable value of $D$, denoted by $D_{E}$, is determined by looking at the first local minimum of the false nearest neighbors (FNN) under changes in the embedding dimension from $D \rightarrow D+1$.

In the next section, we calculate the embedding parameters of NN3 time series.

\section{RECONSTRUCTED DYNAMICS OF THE TIME SERIES IN NN3 DATABASE}

We have calculated the parameters of $\tau$ and $D_{E}$ for the set of 11 time series, including NN3-101 through NN3-111. It should be mentioned that due to the high nonstationarity of four time series, namely NN3-101, NN3-105, NN3-108 and NN3-109, first order differencing is used for detrending the raw data. By detrending, we mean eliminating the long-term trends of the signal, leaving only short-term oscillations. In fact, for these time series the reconstruction is performed on this secondary signal. Here, the normalized embedding delay is calculated by the autocorrelation function of each time series and the first point bellow zero zone is considered as the value of $\tau$. Although, the method of average mutual information (AMI) is more reliable; however, it requires more historical data that is not available in our experiments. The embedding dimension is also calculated by the method of false nearest neighbors (FNN). We have depicted the autocorrelation and[15] FNN function for the time series NN3-106 in Fig. 2 and Fig. 3 respectively. The complete results for all of the time series in the database are reported in Table I.

\section{ALGORITHM IMPLEMENTATION}

To generate the prediction of the next 18 observation, NAR networks are used to model the functions $f$ defined in (7). The reconstructed state space of each data set is used here as the endogenous inputs. The reconstruction vectors $\mathbf{y}_{R}(t)$ defined by (5) is of dimension $D_{E}$. Furthermore, the size of output delay line memory required to perform the embedding, is $\tau \times D_{E}$. However, the delay line memory is only required to provide $D_{E}$ outputs. Therefore, we use $\tau$ equally spaced taps, representing spars connections to the nonlinear structure of NAR. The embedding parameters as well as the nonlinear structures, which are used for different time series in NN3 database are mentioned in Table I.

As the training sets of the given time series are extremely 


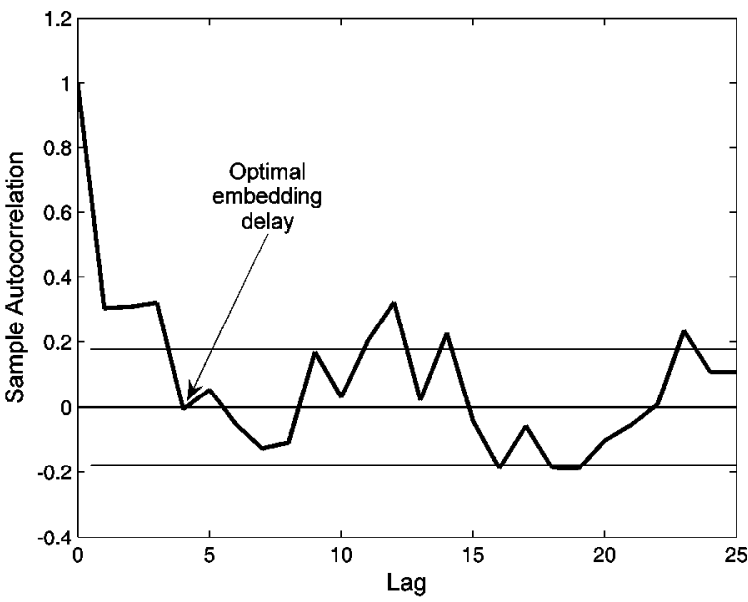

Fig. 2. The autocorrelation function of time series NN3-106. The first point bellow zero zone is considered as the value of normalized embedding delay.

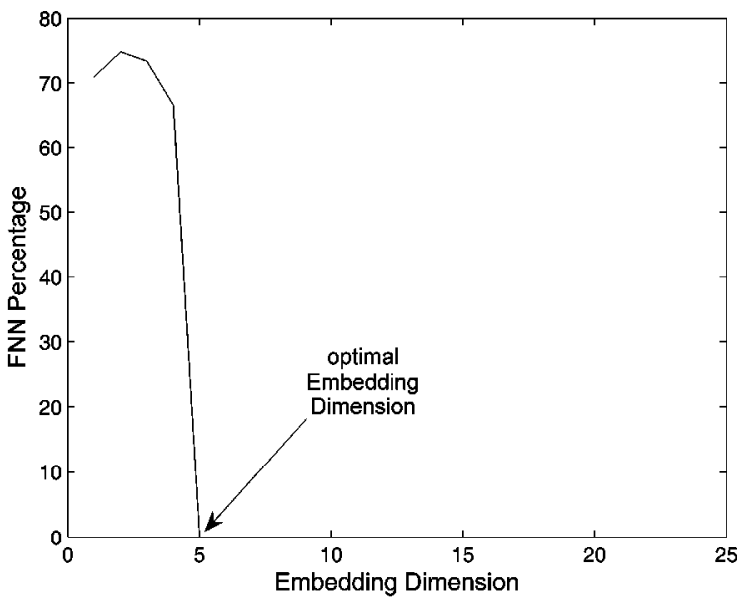

Fig. 3. The percentage of false nearest neighbors for data points of NN3106 time series. The location of the first minimum is reported as the minimum acceptable value of embedding dimension.

TABLE I

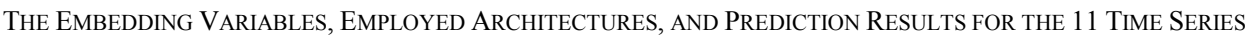

\begin{tabular}{|c|c|c|c|c|c|}
\hline Time Series & $\boldsymbol{\tau}$ & $D_{\mathbf{r}}$ & Network & Train NMSE & $\begin{array}{c}\text { Validation } \\
\text { NMSSE }\end{array}$ \\
\hline NN3-101 & 3 & 4 & {$\left[\begin{array}{llll}\operatorname{De} & 3 & 5 & 1\end{array}\right]$} & 0.049531 & 0.077242 \\
\hline NN3-102 & 6 & 4 & [De 5 1] & 0.042137 & 0.119053 \\
\hline NN3-103 & 6 & 8 & [De $\left.6 \begin{array}{lll}6 & 2 & 1\end{array}\right]$ & 0.058877 & 0.94272 \\
\hline NN3-104 & 6 & 4 & {$\left[\begin{array}{llll}\operatorname{De} & 5 & 3 & 1\end{array}\right]$} & 0.002938 & 0.025725 \\
\hline NN3-105 & 3 & 3 & [De 5 1] & 0.130188 & 1.686444 \\
\hline NN3-106 & 4 & 5 & [De $\left.\begin{array}{llll}5 & 3 & 1\end{array}\right]$ & 0.016508 & 0.299395 \\
\hline NN3-107 & 4 & 4 & [De $\left.2 \begin{array}{lll}6 & 1\end{array}\right]$ & 0.015089 & 0.851522 \\
\hline NN3-108 & 3 & 3 & [De $\left.6 \begin{array}{lll} & 2 & 1\end{array}\right]$ & 0.426625 & 1.732382 \\
\hline NN3-109 & 4 & 6 & [De 5 1] & 0.181523 & 1.388405 \\
\hline NN3-110 & 5 & 3 & [De 5 1] & 0.052671 & 0.055022 \\
\hline NN3-111 & 3 & 3 & [De 3 1] & 0.114562 & 10.92656 \\
\hline
\end{tabular}

TABLE II

Forecasts Results for the NeXt 18 Data Samples of Each Time Series in NN3 Dataset

\begin{tabular}{|c|c|c|c|c|c|c|c|c|c|c|c|}
\hline Time Soriosit & 101 & 102 & 103 & 104 & 105 & 106 & 107 & 108 & 109 & 110 & 111 \\
\hline$t+1$ & 5236 & 4074 & 3953 & 7441 & 4630 & 4765 & 3782 & 2237 & 3590 & 2773 & 3164 \\
\hline$t+2$ & 5246 & 9107 & 39163 & 7400 & 4731 & 5311 & 3757 & 2837 & 3734 & 2613 & 3513 \\
\hline$t+3$ & 5040 & 9099 & 39350 & 7287 & 4827 & 4777 & 3703 & 3025 & 3702 & 2546 & 2404 \\
\hline$t+4$ & 5219 & 9042 & 32050 & 6925 & 4809 & 4431 & 3640 & 3423 & 3816 & 2494 & 3114 \\
\hline$t+5$ & 5007 & 8945 & 13489 & 6910 & 4861 & 4368 & 3708 & 3066 & 3894 & 2539 & 3138 \\
\hline$t+6$ & 5185 & 8744 & 4586 & 3438 & 5015 & 4673 & 3804 & 3195 & 3901 & 2121 & 2521 \\
\hline$t+7$ & 5067 & 8440 & 4127 & 3079 & 4974 & 4305 & 3763 & 4285 & 3804 & 2341 & 2496 \\
\hline$t+8$ & 4847 & 7967 & 3928 & 3630 & 4962 & 4476 & 3804 & 3343 & 3968 & 2650 & 2333 \\
\hline$t+9$ & 5261 & 7580 & 3921 & 6571 & 5117 & 5284 & 3777 & 3358 & 3914 & 2711 & 3163 \\
\hline$t+10$ & 5037 & 7219 & 4251 & 7175 & 5092 & 4190 & 3760 & 3710 & 3877 & 2667 & 2507 \\
\hline$t+11$ & 5109 & 6848 & 4552 & 7231 & 5044 & 5379 & 3795 & 3398 & 3927 & 3038 & 2591 \\
\hline$t+12$ & 5036 & 6282 & 4111 & 7408 & 5125 & 5172 & 3821 & 3419 & 3965 & 2839 & 2854 \\
\hline$t+13$ & 5146 & 5732 & 4099 & 7400 & 5133 & 4771 & 3786 & 3146 & 3945 & 2575 & 3076 \\
\hline$t+14$ & 5116 & 9078 & 36065 & 7410 & 5084 & 5403 & 3759 & 3562 & 3988 & 2541 & 3180 \\
\hline$t+15$ & 4923 & 9114 & 36168 & 7315 & 5082 & 4771 & 3759 & 3637 & 4147 & 2563 & 2427 \\
\hline$t+16$ & 5139 & 9118 & 31001 & 7146 & 5129 & 4799 & 3772 & 3043 & 4109 & 2492 & 2859 \\
\hline$t+17$ & 4922 & 9099 & 7046 & 7028 & 5116 & 4443 & 3759 & 3783 & 4209 & 2501 & 2817 \\
\hline$t+18$ & 5076 & 9041 & 4151 & 3702 & 5048 & 4747 & 3775 & 3927 & 4322 & 2615 & 2776 \\
\hline
\end{tabular}


small, we have just considered $10 \%$ of each data set as validation data. The validation data in these experiments play a very important role. As the desired values of the time series are not available, to achieve the best results, we have trained many NAR networks in each case. Then, the network with the best performance over validation data is used to generate the forecasts for the next 18 data samples.

\section{FORECASTING RESULTS}

The normalized mean square error (NMSE) values of the predictions are reported in Table I. Furthermore, the forecasted time series are presented in Table II. As the desired outputs of each network are not available yet, we have not been able to do any analysis on the results.

\section{REFERENCES}

[1] S. Haykin, Neural Networks: A Comprehensive Foundation, Englewood Cliffs, NJ: Prentice Hall, 2nd ed., 1999, ch. 14.

[2] F. Takens, "On the numerical determination of the dimension of an attractor," in D. Rand, and L. S. Young, eds., Dynamical Systems and Turbulence, Annual Notes in Mathematics, vol. 898, pp.366-381, Berlin: Springer-Verlag.

[3] A. Andalib, S. E. Safavieh, and F. Atry " $\tau$-Step Ahead Forecasts for the Hourly Ontario Energy Price: A Comparative Study, A Critical Analysis of Current One-Step Ahead Forecasts," IEEE Trans. Power Syst., , submitted for publication

[4] A. Andalib, A. R. Sharafat, M. R. Zakeri Nasab, and S. E. Safavieh, "Optimal embedding of NARX networks for learning long-term dependencies in noisy and nonstationary time series," IEEE Trans. Neural Netw., submitted for publication.
[5] Y. Bengio, P. Simard, P. Frasconi, "Learning long-term dependencies with gradient descent is difficult," IEEE Trans. Neural Netw., vol. 5, no. 10, pp. 157-166, Mar. 1994.

[6] T. N. Lin, B. G. Horne, P. Tino, and C. L. Giles, "Learning long-term dependencies in NARX recurrent neural networks," IEEE Trans. Neural Netw., vol.7, no. 6, pp.1329-1337, Nov. 1996.

[7] T. N. Lin, B. G. Horne, and C. L. Giles, "How memory order effect the performance of NARX networks," Inst. Adv. Comput. studies, Univ. Maryland, Collage Park, Tech. Rep. UMIACS-TR-96-76 and CS-TR-3706, 1996.

[8] G. Box, G. M. Jenkins, Time Series Analysis: Forecasting and Control, second edition. Oakland, CA: Holden-Day, 1976.

[9] F. Takens, "Detecting strange attractors in fluid turbulence" in D. Rand, and L. S. Young, eds., Dynamical Systems and Turbulence, Berlin: Springer-Verlag.

[10] A. M. Albano, J. Muench, C.Schwartz, A. I. Mees, P.E. Rapp. "Singular value decomposition and the Grassberger-Procaccia algorithm". Phys. Rev. A, 38:3017-3026, 1988.

[11] D. S. Broomhead, G. P. King, "On the qualitative analysis of experimental dynamical systems", In S. Sarker, editor, Nonlinear phenomena and mental chaos, page113. Adam Hilger, Bristol, 1986.

[12] W. Liebert, H. Schuster, "Proper choice of the time delay for the analysis of chaotic time series" Physics Letters A, Volume 142, Issue 2-3, p. 107-111.

[13] A. M. Fraser, H. L. Swinny. "Independent coordinates for strange attractors from mutual information". Phys. Rev. A, 33:1134-1140, 1986.

[14] T. Buzug, G. Pfister, "Optimal delay time and embedding dimension for delay-time coordinates by analysis of the global and local behavior of strange attractors", Phys. Rev. A. 45:7073-7084, 1992

[15] M. B. Kennel, R. Brown, and H. D. I. Abarbanet, "Determining embedding dimension for phase-space reconstruction using a geometrical construction," Phys. Rev. A 45, pp. 3403-3411, 1992. 